08

\title{
Формирование наночастиц натрия и калия при локальном электронном облучении щелочно-галоидных кристаллов
}

\author{
() А.И. Сидоров, ${ }^{1}$ У.В. Юрина, ${ }^{2}$ О.А. Подсвиров ${ }^{2}$ \\ ${ }^{1}$ Университет ИТМО, \\ 197101 Санкт-Петербург, Россия \\ ${ }^{2}$ Санкт-Петербургский политехнический университет Петра Великого, \\ 195251 Санкт-Петербург, Россия \\ e-mail: sidorov@oi.ifmo.ru
}

Поступило в Редакцию 3 января 2019г.

В окончательной редакции 3 января 2019г.

Принято к публикации 4 фревраля 2019г.

\begin{abstract}
Экспериментально показано, что при локальном облучении кристаллов $\mathrm{NaCl}, \mathrm{KCl}$ и $\mathrm{KBr}$ электронами с энергией $50 \mathrm{keV}$ в них формируются сферические металлические наночастицы натрия и калия, обладающие плазмонными резонансами в видимой области спектра. Представлены спектры оптической плотности кристаллов после облучения электронами с разной дозой. Методами численного моделирования изучены вероятность нахождения наночастиц в твердой или жидкой фазе, а также интерференционные эффекты, возникающие в облученной зоне кристаллов.
\end{abstract}

DOI: 10.21883/JTF.2019.07.47803.2-19

\section{Введение}

Оптические материалы с наночастицами металлов находят применение в качестве цветных оптических фильтров, нелинейно-оптических сред [1-5] и сред для записи оптической информации [6-8]. В ряде работ теоретически показано, что прозрачные среды с наночастицами металлов могут обладать свойствами метаматериалов, и на их основе могут быть созданы суперлинзы [9-12]. Для формирования наночастиц металлов в оптических материалах может быть использован ряд методов. Наночастицы металлов могут быть сформированы в стеклах, содержащих ионы металла, непосредственно при его синтезе в восстановительных условиях [13], методом ионной имплантации [14] или ионного обмена [15] с последующей термообработкой, методом фототермоиндуцированной кристаллизации [13,16], лазерным облучением с последующей термообработкой стекла [6], а также электронно-лучевым методом [17-21]. Достоинством последнего метода является то, что наночастицы металла диаметром 5-10 nm могут быть сформированы в стекле локально, в том числе в нанометровых масштабах.

Основной особенностью локального облучения диэлектриков электронами с относительно низкой энергией $(E=5-100 \mathrm{keV})$ является то, что электроны теряют энергию и накапливаются в тонком приповерхностном слое материала, ограниченном по трем координатам. При этом под поверхностью формируется область отрицательного заряда, образованная термализованными электронами. Как теоретически показано в [22], заряд в этой области располагается слоями, параллельными поверхности. Причиной этого является различие в подвижности положительных и отрицательных носителей заряда (например, электронов и дырок или подвижных положительных ионов). Напряженность электрического поля в этих слоях может достигать $100 \mathrm{kV} / \mathrm{cm}$ [22]. В результате этого происходит полевая миграция подвижных положительных ионов из объема материала в область отрицательного заряда, где эти ионы восстанавливаются термализованными электронами. Таким образом, локальное электронное облучение приводит к существенному перераспределению компонентов материала и увеличению концентрации подвижных компонентов в тонком слое вблизи поверхности. Экспериментально это было подтверждено в работе [18] на примере силикатного стекла с серебром. Данный метод позволяет формировать тонкие приповерхностные композитные слои с металлическими наночастицами, которые могут обладать свойствами плазмонных волноводов и других плазмонных наноструктур, метаповерхностей, нанопроводников электрического тока, а также записывать оптическую информацию в субмикронных масштабах.

Как правило, в оптических материалах синтезируют наночастицы благородных металлов $(\mathrm{Ag}, \mathrm{Au}, \mathrm{Pt})$ и меди. В то же время наночастицы щелочных металлов, имеющие плазмонные резонансы в видимой области спектра [23], также представляют практический интерес для создания нелинейно-оптических сред и сред для записи оптической информации. В работах [24-26] показано, что при $\gamma$-облучении кристаллов $\mathrm{LiF}$ и последующей термообработке в них формируются металлические наночастицы $\mathrm{Li}$, обладающие плазмонным резонансом на длине волны $550 \mathrm{~nm}$. В кристаллах $\mathrm{NaF}$ аналогичным методом могут быть сформированы наночастицы $\mathrm{Na}$ [26]. В работе [27] показано, что при синтезе натриевосодержащих фторфосфатных стекол в восстановительных условиях в стекле формируются наночастицы натрия. В работе [28] экспериментально показано, что 
сферические наночастицы натрия, обладающие плазмонным резонансом на длине волны $\lambda=405-410 \mathrm{~nm}$, могут быть сформированы в натриево-силикатном стекле с помощью электронного облучения и последующей термообработки выше температуры стеклования. Методами численного моделирования в настоящей работе установлено, что наночастицы состоят из сферического ядра из твердого (возможно, жидкого) натрия, и содержат оболочку из оксида натрия, окруженную внешней вакуумной или газовой оболочкой. При локальном электронном облучении монокристаллов фторидов в них могут быть сформированы металлические наночастицы $\mathrm{Li}, \mathrm{Mg}$, Са и Ва $[29,30]$. Причем плазмонные резонансы наночастиц $\mathrm{Mg}$ имеют форму, характерную для плазмонных резонансов вытянутых сфероидов [30].

Целями настоящей работы было экспериментальное исследование возможности синтеза наночастиц натрия и калия в монокристаллах $\mathrm{NaCl}, \mathrm{KCl}$ и $\mathrm{KBr}$ методом локального электронного облучения, а также изучение особенностей структуры и других особенностей таких наночастиц методами оптической спектроскопии и численного моделирования.

\section{Методики экспериментов и численного моделирования}

В экспериментах использовались полированные пластины монокристаллов $\mathrm{NaCl}, \mathrm{KCl}$ и $\mathrm{KBr}$ толщиной 3-4 mm. Облучение электронами проводилось на сканирующем электронном микроскопе JEBD-2 с энергией электронов $E=50 \mathrm{keV}$. Плотность электронного тока составляла $j=50 \mu \mathrm{A} / \mathrm{cm}^{2}$, доза электронного облучения $Q$ варьировала в диапазоне $10-100 \mathrm{mC} / \mathrm{cm}^{2}$. Расчет показывает, что для указанной энергии электронов максимум их энергетических потерь находится на глубине $10-15 \mu \mathrm{m}$ в зависимости от материала монокристалла. Диаметр облученной зоны определялся удобством оптических измерений и был равен $2 \mathrm{~mm}$. Для удаления поверхностного заряда при облучении образцы покрывались слоем $\mathrm{Al}$ толщиной $50 \mathrm{~nm}$, который после облучения удалялся. Образцы облучались при комнатной температуре, однако расчет показывает, что при $E=50 \mathrm{keV}$ и $j=50 \mu \mathrm{A} / \mathrm{cm}^{2}$ приповерхностный слой кристалла толщиной $1 \mu \mathrm{m}$ нагревается до $180-190^{\circ} \mathrm{C}$. Температуры плавления $\mathrm{Na}$ и К равны 97.8 и $63.5^{\circ} \mathrm{C}$ соответственно [31]. Очевидно, что наночастицы $\mathrm{Na}$ и $\mathrm{K}$, формирующиеся в процессе облучения, исходно находятся в жидкой фазе. Можно предположить, что и после облучения они могут находиться в жидкой фазе в виде переохлажденной жидкости.

В связи с высокой химической активностью щелочных металлов приготовление образцов для получения ТЕМизображений (Transmission Electron Microscopy) не представлялось возможным. Поэтому измерения после облучения проводились методами оптической спектроскопии.
Спектры оптической плотности измерялись на спектрофотометре Lambda 650 (Perkin Elmer) в диапазоне длин волн 300-800 nm с шагом $1 \mathrm{~nm}$ при комнатной температуре.

Численное моделирование сечений поглощения наночастиц $\sigma_{a}$ проводилось в дипольном квазистатическом приближении [32,33], которое справедливо для наночастиц, имеющих размер много меньший длины волны (как правило, менее $20 \mathrm{~nm}$ ). Сечение поглощения наночастицы определяется выражением [32]

$$
\sigma_{a}=k \operatorname{Im}(\delta),
$$

где $\delta-$ поляризуемость наночастицы, $k-$ волновое число $(k=2 \pi / \lambda)$.

Поляризуемость сферической наночастицы может быть определена по формуле [32]

$$
\delta=4 \pi r^{3} \frac{\varepsilon_{p}-\varepsilon_{h}}{\varepsilon_{p}+2 \varepsilon_{h}},
$$

где $\varepsilon_{h}, \varepsilon_{p}$ - диэлектрические проницаемости окружающей среды и наночастицы соответственно; $r$ - радиус наночастицы. При моделировании радиус наночастиц принимался равным $5 \mathrm{~nm}$.

Как будет показано ниже, наиболее реалистичной моделью в нашем случае является металлическая наночастица с диэлектрической оболочкой. Для сферической наночастицы с оболочкой выражение для поляризуемости имеет вид [32]

$$
\begin{gathered}
\delta=4 \pi r_{s}^{3} \frac{\varepsilon_{s} \varepsilon_{a}-\varepsilon_{h} \varepsilon_{b}}{\varepsilon_{s} \varepsilon_{a}+2 \varepsilon_{h} \varepsilon_{b}}, \\
\varepsilon_{a}=\varepsilon_{c}(3-2 P)+2 \varepsilon_{s} P, \\
\varepsilon_{b}=\varepsilon_{c}+\varepsilon_{s}(3-P), \quad P=1-\left(\frac{r_{c}}{r_{s}}\right)^{3} .
\end{gathered}
$$

Здесь $\varepsilon_{h}, \varepsilon_{c}, \varepsilon_{s}$ - диэлектрические проницаемости среды, ядра и оболочки наночастицы соответственно; $r_{c}$ радиус ядра; $r_{s}-$ радиус оболочки. Условием возникновения плазмонного резонанса является обращение в нуль действительной части знаменателя поляризуемости наночастицы [33].

При электронном облучении металлические наночастицы образуют под поверхностью кристалла тонкий композитный слой, в котором может происходить интерференция. Расчет оптических свойств трехслойной интерференционной системы с композитным слоем, содержащим металлические наночастицы, проводился по формулам Френеля.

Для оценки фактора заполнения композитного слоя наночастицами с оболочкой использовался метод „гомогенизации“ [34]. Для этого вводилась эквивалентная наночастица без оболочки и определялась ее эффективная диэлектрическая проницаемость $\epsilon$ с учетом размерных эффектов [34,35] на основании теорий М. Гарнетта и Дж. Ми:

$$
\frac{\epsilon-1}{(\epsilon+2)-(\epsilon-1) x^{2}-(\epsilon-1) x^{3} 2 i / 3}=\frac{3 i a_{1}}{2 x^{3}} p .
$$


Здесь $p=r c^{3} / r s^{3} ; a_{1}$ - дипольный электрический коэффициент из теории Дж. Ми, связанный с поляризуемостью наночастицы выражением:

$$
\delta=\frac{3 i a_{1}}{2 x^{3}}, \quad x=\frac{2 \pi r_{s}}{\lambda},
$$

где $\lambda$ - длина волны излучения.

Затем определялся фактор заполнения $f$ композитного слоя с эквивалентными наночастицами с использованием теории М. Гарнетта:

$$
\frac{\varepsilon \mathrm{eff}-\varepsilon_{h}}{\varepsilon_{\mathrm{eff}}+2 \varepsilon_{h}}=f \frac{\epsilon-\varepsilon_{h}}{\epsilon+2 \varepsilon_{h}} .
$$

Здесь $\varepsilon_{\mathrm{eff}}$ - эффективная диэлектрическая проницаемость композитного слоя.

При численном моделировании использовались оптические константы $\mathrm{NaCl}, \mathrm{KCl}$ и $\mathrm{KBr}$ из [36,37]. Дисперсия оптических констант $\mathrm{Na}$ и $\mathrm{K}$ в твердой и жидкой фазах задавалась по данным, приведенным в [36-39].

\section{Экспериментальные результаты}

На рис. 1 преведены спектры оптической плотности кристалла $\mathrm{NaCl}$ до и после электронного облучения. Из рисунка видно, что облучение электронами приводит к появлению полосы поглощения в спектральном интервале $350-550 \mathrm{~nm}$ с максимумом на $\lambda=450 \mathrm{~nm}$. Увеличение дозы облучения приводит к увеличению амплитуды данной полосы поглощения. На спектре также появляются две полосы поглощения с максимумами на

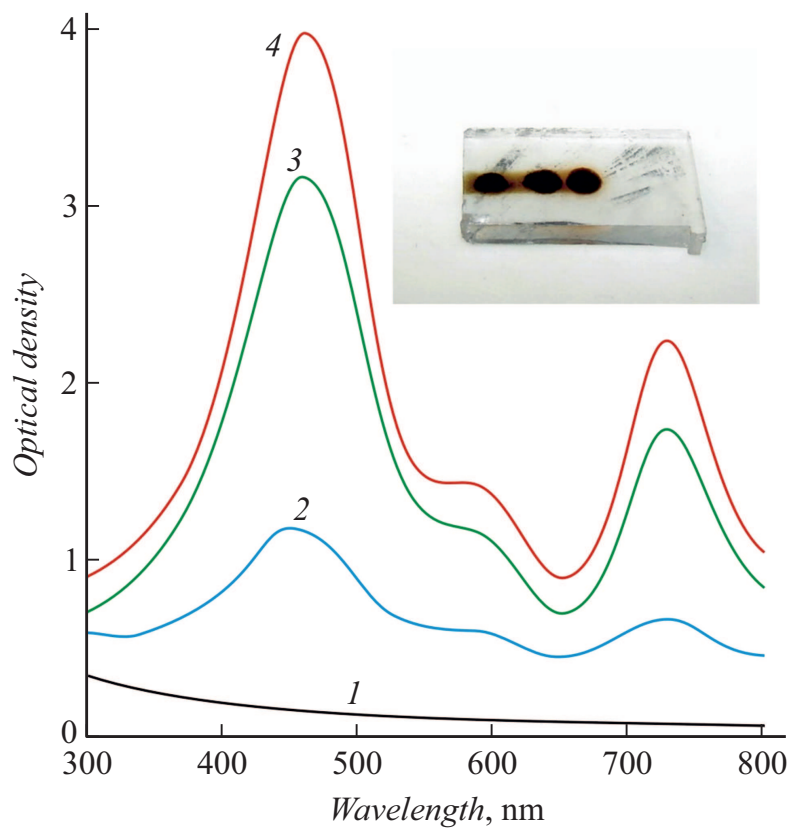

Рис. 1. Спектры оптической плотности монокристалла $\mathrm{NaCl}$ до $(1)$ и после $(2,3)$ электронного облучения: $2-Q=10$, $3-50,4-100 \mathrm{mC} / \mathrm{cm}^{2}$. На вставке - фотография кристалла $\mathrm{NaCl}$ после электронного облучения.

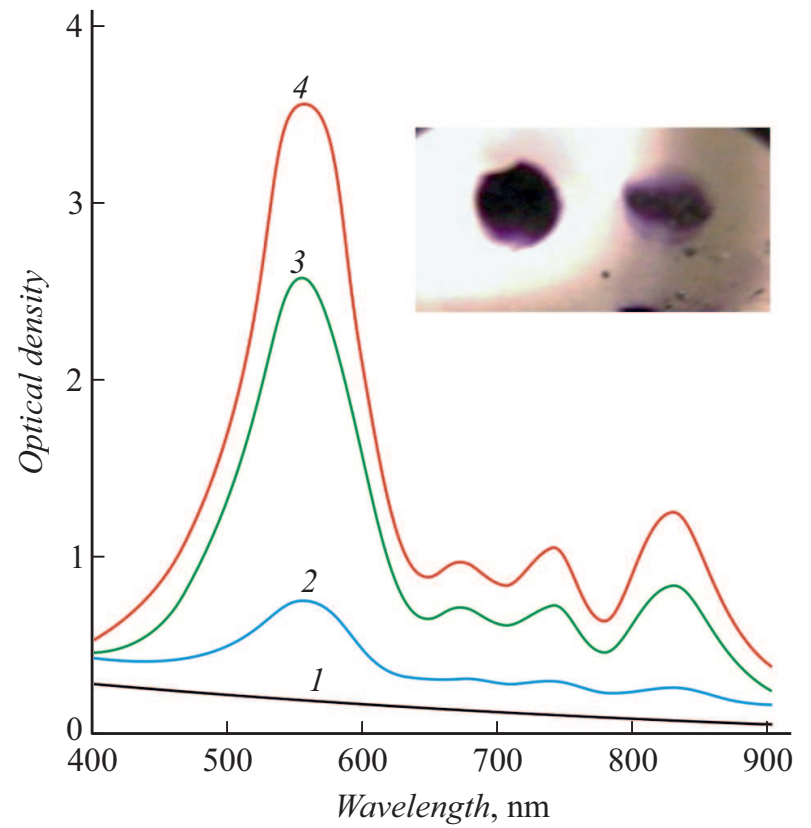

Pис. 2. Спектры оптической плотности монокристалла $\mathrm{KCl}$ до $(1)$ и после $(2,3)$ электронного облучения: $2-Q=10$, $3-50,4-100 \mathrm{mC} / \mathrm{cm}^{2}$. На вставке - фотография кристалла $\mathrm{KCl}$ после электронного облучения.

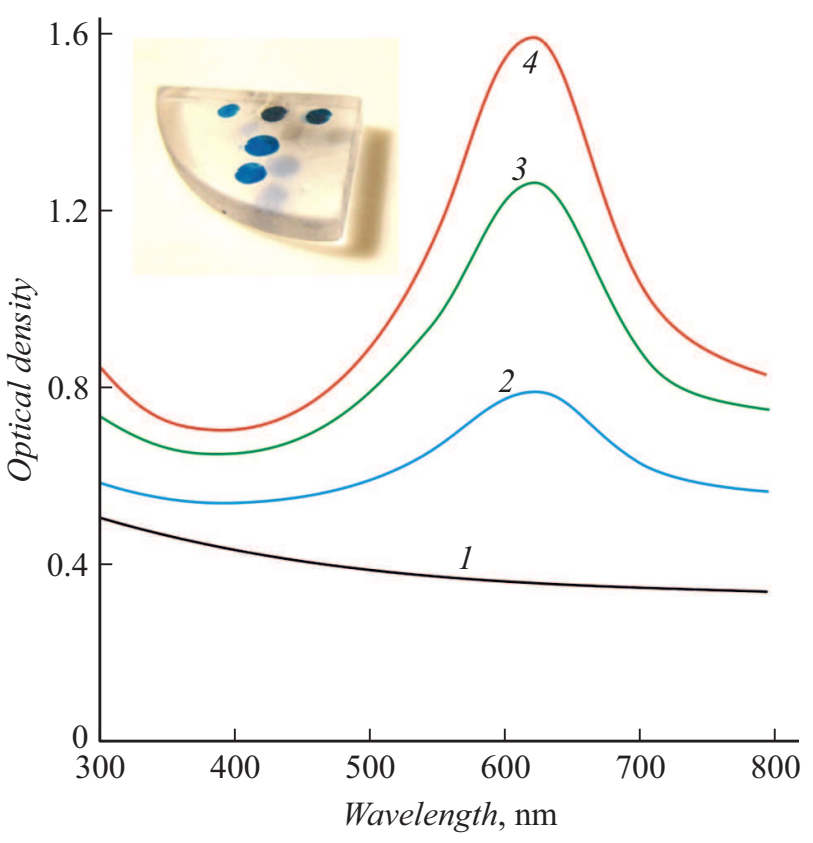

Pис. 3. Спектры оптической плотности монокристалла $\mathrm{KBr}$ до $(1)$ и после $(2,3)$ электронного облучения: $2-Q=10$, $3-50,4-100 \mathrm{mC} / \mathrm{cm}^{2}$. На вставке - фотография кристалла $\mathrm{KBr}$ после электронного облучения.

$\lambda=580$ и $730 \mathrm{~nm}$, амплитуда которых также увеличивается при увеличении дозы облучения. В облученной зоне кристалла появляется окраска, цвет которой изменяется от темно-коричневого до черного (см. вставку на 


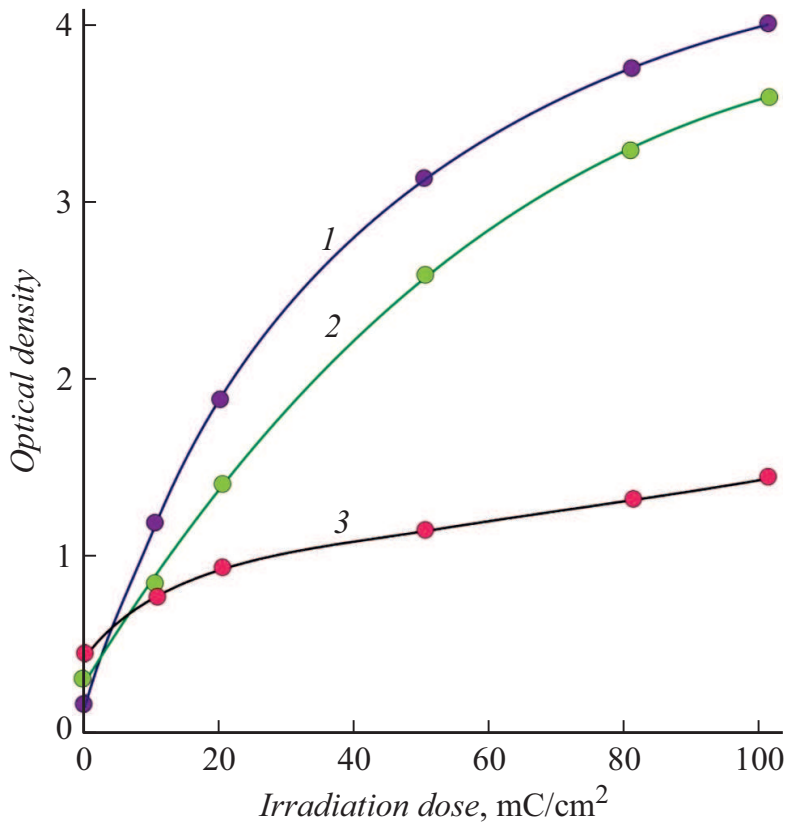

Pис. 4. Зависимость оптической плотности в максимуме основной полосы поглощения от дозы электронного облучения: $1-\mathrm{NaCl}(\lambda=450 \mathrm{~nm}), 2-\mathrm{KCl}(\lambda=550 \mathrm{~nm}), 3-\mathrm{KBr}$ $(\lambda=610 \mathrm{~nm})$.

рис. 1). Из вставки также видно, что изменение окраски происходит и в области перемещения электронного луча между зонами облучения. Это указывает на то, что даже при дозах облучения, меньших $1 \mathrm{mC} / \mathrm{cm}^{2}$, в приповерхностной области кристалла происходят заметные изменения. Учитывая малую толщину поглощающего слоя, можно оценить коэффициент поглощения в максимуме кривой $4(\lambda=450 \mathrm{~nm})$, как превышающий $1000 \mathrm{~cm}^{-1}$.

На рис. 2 приведены спектры оптической плотности кристалла $\mathrm{KCl}$ до и после электронного облучения. Как видно из рисунка, облучение электронами приводит к появлению полосы поглощения в спектральном интервале 450-650 nm с максимумом на $\lambda=550 \mathrm{~nm}$, а также трех дополнительных полос с максимумами на $\lambda=660$, 730 и $830 \mathrm{~nm}$. Увеличение дозы облучения приводит к увеличению амплитуды этих полос поглощения. В облученной зоне кристалла появляется окраска, цвет которой изменяется от светло- до темно-сиреневого (см. вставку в рис. 2).

Локальное электронное облучение кристаллов $\mathrm{KBr}$ также приводит к появлению интенсивной и широкой полосы поглощения в облученной зоне (рис. 3). Данная полоса расположена в спектральном интервале $450-700 \mathrm{~nm}$ и имеет максимум на $\lambda=610 \mathrm{~nm}$. Дополнительные полосы поглощения в данном случае отсутствуют. Амплитуда полосы поглощения увеличивается при увеличении дозы электронного облучения. В облученной зоне кристалла появляется окраска, цвет которой изменяется от светло- до темно-синего (см. вставку в рис. 3).
Зависимости оптической плотности в максимуме основной полосы поглощения от дозы электронного облучения для кристаллов $\mathrm{NaCl}, \mathrm{KCl}$, и $\mathrm{KBr}$ показаны на рис. 4. Из рисунка видно, что на начальных участках зависимостей увеличение дозы облучения приводит к резкому увеличению оптической плотности. При $Q>30 \mathrm{mC} / \mathrm{cm}^{2}$ зависимости становятся более пологими. Для кристалла $\mathrm{NaCl}$ при увеличении дозы облучения от 0 до $100 \mathrm{mC} / \mathrm{cm}^{2}$ происходит увеличение оптической плотности в 40 раз, для кристалла $\mathrm{KCl}$ - в 12 раз, для кристалла $\mathrm{KBr}$ - в 3.2 раза. Для всех трех типов кристаллов при их хранении при комнатной температуре окраска и поглощение в облученной зоне сохраняются, как минимум, в течение нескольких месяцев. Термообработка при $300^{\circ} \mathrm{C}$ в течение $1 \mathrm{~h}$ приводит к незначительному уменьшению интенсивности окраски и амплитуды полос поглощения.

\section{Обсуждение результатов}

Как было отмечено во Введении, основными процессами, инициирующими образование наночастиц в облученной зоне, являются формирование области отрицательного заряда вблизи поверхности кристалла, полевая миграция положительных ионов металла в эту область и их восстановление термализованными электронами. Радиационные дефекты, возникающие при электронном облучении, и нагрев приповерхностного слоя кристалла электронным лучом повышают эффективность процесса полевой миграции. В результате в облученной зоне существенно увеличивается концентрация атомов металла. Конкурирующим процессом является ионизация атомов металла быстрыми электронами. Однако возникающие при этом свободные электроны в основном остаются в облученной зоне и могут снова участвовать в процессе восстановления ионов металла. С энергетической точки зрения атомам металла выгодно группироваться в субнаноразмерные молекулярные кластеры $\mathrm{Na}_{n}$ и $\mathrm{K}_{n}$, а затем и в наночастицы, так как потенциальная энергия таких структур по мере увеличения их размеров уменьшается [40]. В результате в облученной зоне кристалла формируются металлические наночастицы, обладающие плазмонным резонансом. Рассмотрим особенности этих наночастиц, следующие из спектрального положения и формы полос поглощения, соответствующих плазмонному резонансу.

Плазмонные полосы поглощения с максимумами на $\lambda=450 \mathrm{~nm}$ для $\mathrm{NaCl}, \lambda=550 \mathrm{~nm}$ для $\mathrm{KCl}$ и $\lambda=610 \mathrm{~nm}$ для $\mathrm{KBr}$ симметричны, что указывает на то, что наночастицы имеют сферическую форму, а их размеры много меньше длины волны. В то же время численное моделирование показывает, что спектральное положение их полос поглощения смещено в коротковолновую область спектра по сравнению с плазмонными резонансами наночастиц, окруженных сплошным идеальным кристаллом соответствующего состава. На рис. 5 показаны спектры 


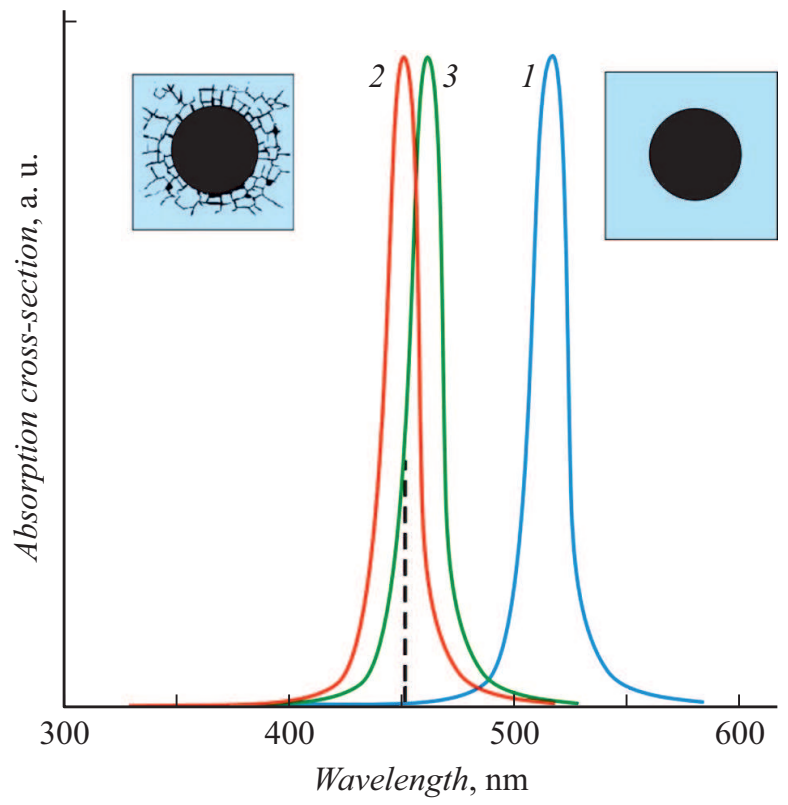

Рис. 5. Расчетные нормированные спектры сечения поглощения сферических наночастиц $\mathrm{Na}$ в кристалле $\mathrm{NaCl}: 1-$ твердый $\mathrm{Na}$ в идеальном кристалле, 2 - твердый $\mathrm{Na}$ в оболочке из дефектов кристалла, 3 - жидкий Na в оболочке из дефектов кристалла. Штрих - спектральное положение максимума плазмонного резонанса, полученное в эксперименте. На вставках — геометрия наночастиц.

сечения поглощения сферической наночастицы $\mathrm{Na}$ в матрице $\mathrm{NaCl}$ (кривая 1) и наночастицы $\mathrm{Na}$ с оболочкой, имеющей показатель преломления меньший, чем показатель преломления $\mathrm{NaCl}$ (кривая 2). Из рисунка видно, что при показателе преломления оболочки $n_{s}=1.4$ расчетный максимум плазмонного резонанса совпадает с экспериментальным результатом. При моделировании толщина оболочки была выбрана равной $7 \mathrm{~nm}$. Дальнейшее увеличение толщины оболочки практически не влияет на спектральное положение плазмонного резонанса, так как в этом случае наночастица „воспринимает“ оболочку как протяженную окружающую среду. Появление оболочки с низким показателем преломления вызвано образованием большого количества кристаллических дефектов вокруг наночастицы из-за уменьшения концентрации ионов $\mathrm{Na}$ в кристаллической решетки. Второй причиной образования оболочки является появление нанотрещин в кристалле вокруг наночастицы из-за локальных механических напряжений, возникающих при росте наночастицы (см. вставку в рис. 5). Спектральное уширение плазмонной полосы, измеренной в эксперименте, связано с разбросом показателей преломления и толщины оболочек вокруг наночастиц $\mathrm{Na}$ в облученной зоне кристалла, а также с отклонением их формы от сферической.

Так как существует вероятность того, что при электронном облучении в стекле формируются наночастицы $\mathrm{Na}$, находящиеся в жидкой фазе, было проведено числен- ное моделирование сечения поглощения сферической жидкой наночастицы $\mathrm{Na}$ в кристалле $\mathrm{NaCl}$ с оболочкой, описанной выше. Результат показан на рис. 5, кривая 3. Из рисунка видно, что в этом случае происходит незначительное длинноволновое смещение плазмонной полосы поглощения. Данный эффект выражен слабо, так как оптические константы Na в твердой и жидкой фазах отличаются незначительно. Поскольку экспериментально измеренная плазмонная полоса поглощения наночастиц $\mathrm{Na}$ имеет значительную спектральную ширину, ответить на вопрос, в твердой или жидкой фазе находятся наночастицы $\mathrm{Na}$, по результатам оптических измерений не представляется возможным.

Аналогичное численное моделирование было проведено для сферических наночастиц $\mathrm{K}$ в кристаллах $\mathrm{KCl}$ и $\mathrm{KBr}$. Результаты представлены на рис. 6 и 7. Из рисунков видно, что, как и в случае наночастиц $\mathrm{Na}$, с экспериментальными результатами согласуется сечение поглощения наночастиц с оболочкой, имеющей низкий показатель преломления. Для $\mathrm{KCl}$ наилучшее согласование реализуется при $n_{s}=1.3$, для $\mathrm{KBr}-$ при $n_{s}=1.36$. Из рисунков также видно, что численное моделирование не позволяет достоверно определить, в твердой или жидкой фазе находятся наночастицы К.

Рассмотрим возможную причину возникновения особенностей в длинноволновой части спектров оптической плотности, показанных на рис. 1 и 2. При облучении электронами кристаллов $\mathrm{NaCl}, \mathrm{KCl}$ и $\mathrm{KBr}$ в них возникают радиационные дефекты, такие как F-, $\mathrm{F}_{2-}$ и $\mathrm{F}_{3}$-центры [41]. Однако спектральное положение

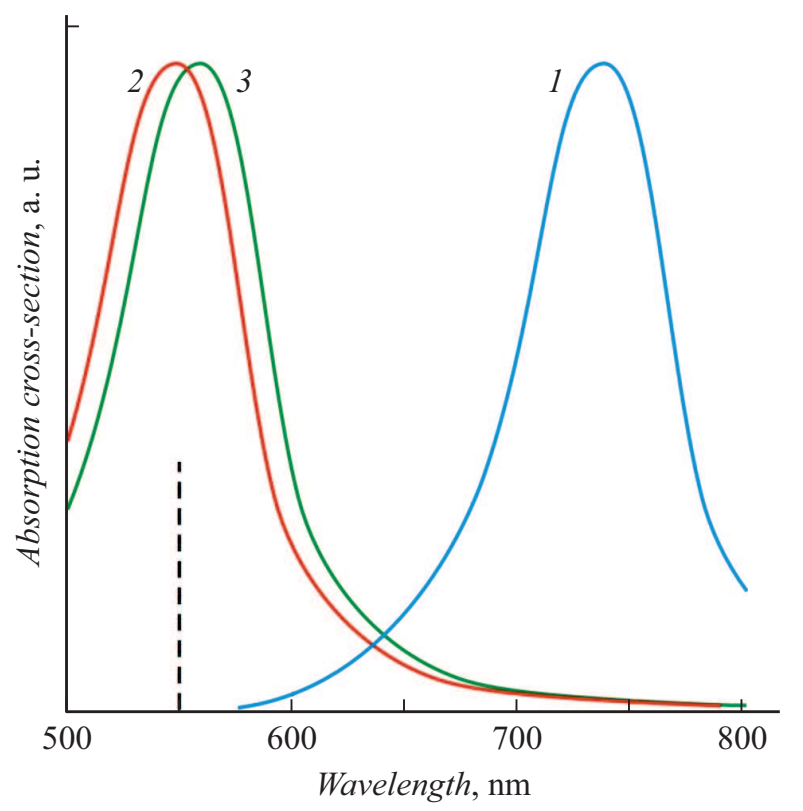

Рис. 6. Расчетные нормированные спектры сечения поглощения сферических наночастиц $\mathrm{K}$ в кристалле $\mathrm{KCl}: 1$ твердый К в идеальном кристалле, 2 - твердый К в оболочке из дефектов кристалла, 3 - жидкий $\mathrm{K}$ в оболочке из дефектов кристалла. Штрих - спектральное положение максимума плазмонного резонанса, полученное в эксперименте. 


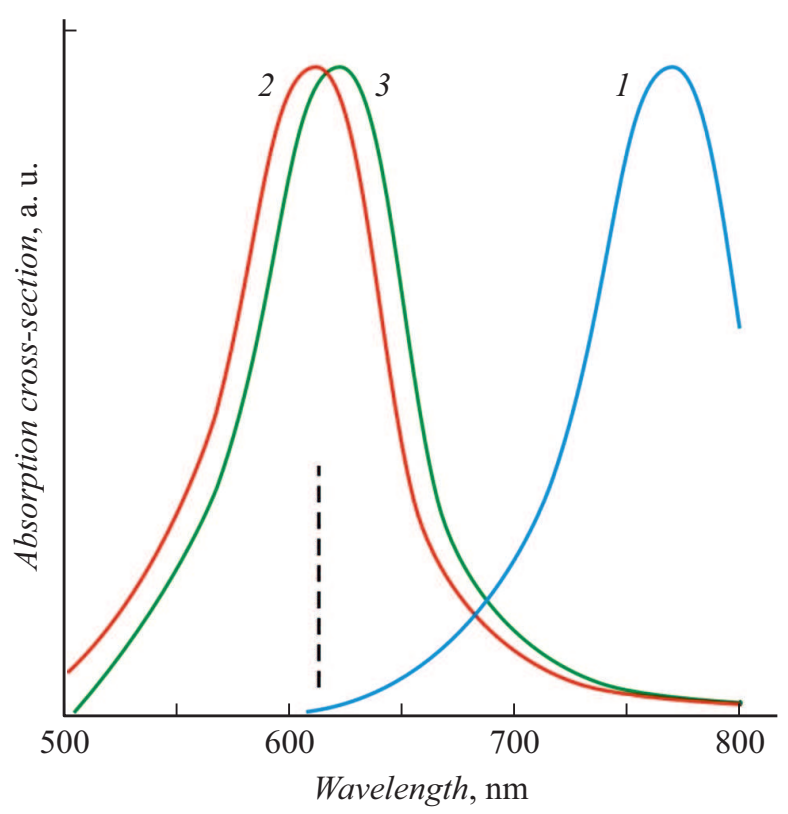

Рис. 7. Расчетные нормированные спектры сечения поглощения сферических наночастиц $\mathrm{K}$ в кристалле $\mathrm{KBr}$ : 1 твердый $\mathrm{K}$ в идеальном кристалле, 2 - твердый $\mathrm{K}$ в оболочке из дефектов кристалла, 3 - жидкий $\mathrm{K}$ в оболочке из дефектов кристалла. Штрих - спектральное положение максимума плазмонного резонанса, полученное в эксперименте.

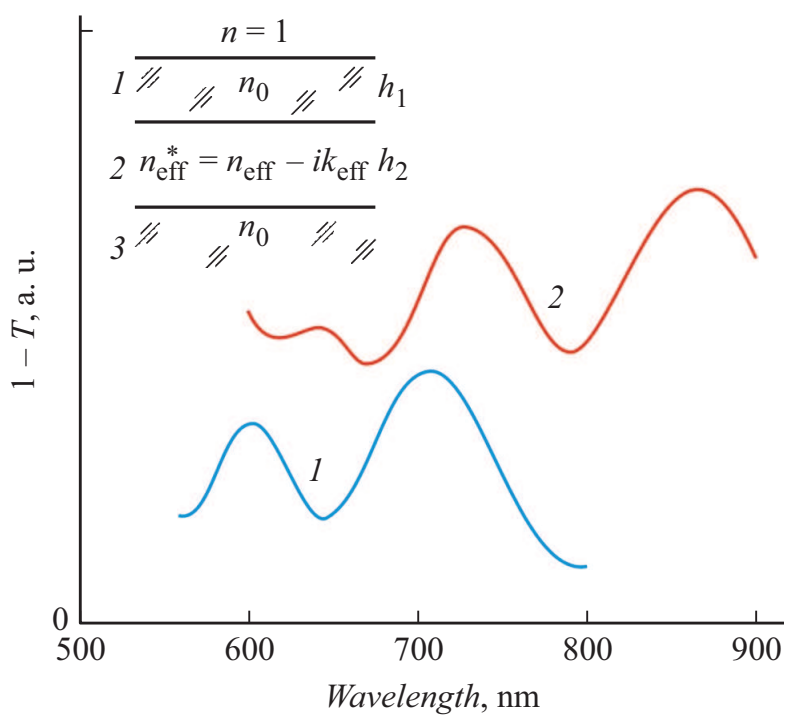

Рис. 8. Расчетные интерференционные спектры трехслойной структуры, показанной на вставке: 1 - для кристалла $\mathrm{NaCl}$, 2 - для кристалла $\mathrm{KCl} ; n_{0}$ - показатель преломления кристалла $\mathrm{NaCl}$ или $\mathrm{KCl}$.

их полос поглощения не совпадает с особенностями спектров, показанных на рис. $1-3$. Кроме того, такие дефекты при комнатной температуре нестабильны [41]. Причиной образования этих спектральных особенностей может быть следующее. При электронном облучении диэлектриков в них формируются тонкие заряженные
Расчетные характеристики трехслойной интерференционной структуры, показанной на вставке в рис. 8

\begin{tabular}{c|c|c|c|c|c}
\hline Кристалл & $n_{\text {eff }}$ & $k_{\text {eff }}$ & $h_{1}, \mu \mathrm{m}$ & $h_{2}, \mu \mathrm{m}$ & $f$ \\
\hline $\mathrm{NaCl}$ & 1.3 & 0.0065 & 0.55 & 1.28 & 0.27 \\
$\mathrm{KCl}$ & 1.2 & 0.0050 & 0.42 & 1.95 & 0.25
\end{tabular}

слои, параллельные облучаемой поверхности и расположенные под ней [22]. Таким же образом располагаются и металлические наночастицы [18]. Так как в композитном слое, содержащем наночастицы, его эффективный комплексный показатель преломления $\left(n_{\mathrm{eff}}^{*}=n_{\mathrm{eff}}-i k_{\mathrm{eff}}\right)$ отличается от показателей преломления окружающих слоев, в такой слоистой структуре может возникать интерференция, приводящая к модуляции коэффициента пропускания $T$. Геометрия такой трехслойной интерференционной структуры показана на вставке в рис. 8 . При моделировании проводилось варьирование толщины слоев и эффективного показателя преломления центрального слоя для обеспечения соответствия спектральных зависимостей $(1-T)$ экспериментальным спектральным зависимостям оптической плотности. Результаты численного моделирования показаны на рис. 8. Расчетные характеристики слоев приведены в таблице.

Сравнивая спектры, показанные на рис. 8, с особенностями на спектрах оптической плотности, показанными на рис. 1 и 2, можно сделать вывод, что эти особенности вызваны интерференционными эффектами в композитном слое, содержащем наночастицы. Интерференционные полосы отсутствуют в спектральном интервале плазмонных резонансов наночастиц $\mathrm{Na}$ и $\mathrm{K}$ (см. рис. 1-3). Причиной этого является высокое поглощение в данных спектральных интервалах, что существенно уменьшает эффективность интерференции. Из таблицы видно, что эффективные показатели преломления композитных слоев с наночастицами, полученные путем оптимизации расчетных спектров интерференции, близки к единице. Причиной этого является низкий показатель преломления металлических $\mathrm{Na}$ и $\mathrm{K}$ в данном спектральном интервале (для $\mathrm{Na} n \approx 0.035$, для $\mathrm{K}$ $n \approx 0.05[35-37])$.

Расчетные величины эффективных показателей преломления и поглощения композитного слоя с наночастицами и величины поляризуемостей наночастиц с оболочкой (3) позволяют оценить фактор заполнения (4), (5) композитного слоя наночастицами. Из таблицы видно, что фактор заполнения $f$ составляет $0.25-0.27$, что является вполне реалистичной оценкой.

\section{Заключение}

Показано, что при локальном облучении электронами монокристаллов $\mathrm{NaCl}, \mathrm{KCl}$ и $\mathrm{KBr}$ в тонком приповерхностном слое кристаллов формируются сферические наночастицы $\mathrm{Na}$ и K, обладающие плазмонными 
резонансами и имеющие оболочки из дефектов кристаллической решетки. Оптические методы не позволяют определить, в каком состоянии, твердом или жидком, находятся наночастицы при комнатной температуре. Эффективный показатель преломления композитного слоя с наночастицами лежит в диапазоне $1.2-1.3$. Низкий эффективный показатель преломления композитного слоя приводит к появлению интерференционных эффектов. Полученные результаты могут быть использованы в устройствах нанофотоники и нелинейной оптики, а также при создании метаматериалов.

\section{Финансирование работы}

Работа выполнена при финансовой поддержке Министерства образования и науки Российской Федерации (Проект 16.1651.2017/4.6).

\section{Список литературы}

[1] Hache F., Ricard D., Flytzanis C. // J. Opt. Soc. Am. B. 1986. Vol. 3. N 12. P. $1647-1655$

[2] Haus J.W., Kalyaniwalla N., Inguwa R. // J. Opt. Soc. Am. B. 1989. Vol. 6. N 4. P. 797-807.

[3] Hamanaka Y., Nakamura A., Omi S. // Appl. Phys. Lett. 1999. Vol. 75. N 12. P. $1712-1714$.

[4] Kyoung M., Lee M. // Opt. Comm. 1999. Vol. 171. N 11. P. $145-148$.

[5] Francois L., Mostafavi M., Belloni J. // Non-Lin. Opt. 2001. Vol. 27. N 1-4. P. 319-329.

[6] Ignatiev A.I., Klyukin D.A., Leontieva V.S., Nikonorov N.V., Shakhverdov T.A., Sidorov A.I. // Opt. Mater. Expr. 2015. Vol. 5. N 7. P. $1635-1646$.

[7] Taylor A.B., Michaux P., Mohsin A.S.M., Chon J.W.M. // Opt. Express. 2014. Vol. 22. P. 13234-13243.

[8] Bellec1 M., Royon A., Bousquet B., Bourhis K., Treguer M., Cardinal T., Richardson M., Canioni L. // Opt. Express. 2009. Vol. 17. P. 10304-10318.

[9] Zhang S., Fan W., Malloy K.J., Brueck S.R.J., Panoiu N.C., Osgood R.M. // J. Opt. Soc. Am. B. 2006. Vol. 23. P. 434-438.

[10] Kildishev A.V., Cai W., Chettiar U.K., Yuan H.-K., Sarychev A.K., Drachev V.P., Shalaev V.M. // J. Opt. Soc. Am. B. 2006. Vol. 23. P. 423-433.

[11] Shi L., Gao L., He S., Li B. // Phys. Rev. B. 2007. Vol. 76. P. 045116-1-6.

[12] Cai W., Genov D.A., Shalaev V.M. // Phys. Rev. B. 2005. Vol. 72. P. 193101-1-4.

[13] Silver nanoparticles / Ed. by D.P. Perez. Vukovar: In-Tech, 2010. 334 p.

[14] Stepanov A.L. // Rev. Adv. Mater. Sci. 2003. Vol. 4. P. 45-50.

[15] Sgibnev E.M., Ignatiev A.I., Nikonorov N.V., Efimov A.M., Postnikov E.S. // J. Non-Cryst. Sol. 2013. Vol. 378. P. 213-226.

[16] Dubrovin V.D., Ignatiev A.I., Nikonorov N.V., Sidorov A.I., Shakhverdov T.A., Agafonova D.S. // Opt. Mater. 2014. Vol. 36. P. 753-759.

[17] Podsvirov O.A., Ignatiev A.I., Nashchekin A.V., Nikonorov N.V., Sidorov A.I., Tsekhomskii V.A., Usov O.A., Vostokov A.V. // Nucl. Inst. Meth. Phys. Res. B. 2010. Vol. 268. P. 3103-3108.
[18] Игнатьев А.И., Нащекин А.В., Неведомский В.М., Подсвиров О.А., Сидоров А.И., Соловьев А.П., Усов О.А. // ЖТФ. 2011. Т. 81. Вып. 5. С. 75-80. [Ignatiev A.I., Naschekin A.V., Nevedomsky D.M., Podsvirov O.A., Sidorov A.I., Soloviev A.P., Usov O.A. // Techn. Phys. 2011. Vol. 56. P. 662-667.]

[19] Подсвиров О.А., Сидоров А.И., Цехомский В.А., Востоков А.В. // ФТТ. 2010. Т. 52. Вып. 9. С. 1776-1779. [Podsvirov O.A., Sidorov A.I., Tsikhomsky V.A., Vostokov A.V. // Phys. Sol. Stat. 2010. Vol. 52. P. 1906-1909.]

[20] Востоков А.В., Верзин И.А., Игнатьев А.И., Подсвиров О.А., Сидоров А.И. // Опт. и спектр. 2010. Т. 109. C. 407-412. [Vostokov A.V., Verzin I.A., Ignat'ev A.I., Podsvirov O.A., Sidorov A.I. // Opt. Spectr. 2010. Vol. 109. N 3. P. 366-371.]

[21] Sidorov A.I., Yurina U.V., Rakhmanova G.R., Shinkarenko M.N., Podsvirov O.A., Fedorov Y.K., Nashchekin A.V. // J. Non-Cryst. Sol. 2018. Vol. 499. P. 278-282.

[22] Touzin M., Goeriot D., Guerret-Piécort C., Juvé D., Tréheux D., Fitting H.-J. // J. Appl. Phys. 2006. Vol. 99. P. 114110.

[23] Kreibig U., Vollmer M. Optical properties of metal clusters. Berlin: Springer-Verlag, 1995. 532 p.

[24] Ворожсейкина Л.Ф. // Известия АН СССР, сер. Физика. 1967. T. 31. C. $1937-1940$.

[25] Назаренко В.Н., Нестеренко О.В., Радченко И.С., Степанкина И.Б. // Вост.-Евр. журн. передовых технол. 2013. T. 63. C. $8-18$.

[26] Брюквина Л.И., Мартынович Е.Ф. // ФТТ. 2012. Т. 54. Вып. 12. C. 2248-2253. [Bryukvina L.I., Martynovich E.F. // Phys. Sol. State. 2012. Vol. 54. N 12. P. 2374-2379.]

[27] Kolobkova E.V., Nikonorov N.V. // Alloys and Comp. 2015. Vol. 637. P. 545-551.

[28] Bochkareva E.S., Nikonorov N.V., Podsvirov O.A., Prosnikov M.A., Sidorov A.I. // Plasmonics. 2016. Vol. 11. P. $241-246$.

[29] Ilina E.A., Sidorov A.I., Yurina U.V., Podsvirov O.A. // Nucl. Instr. Meth. Phys. Res. B. 2017. Vol. 412. P. 28-33.

[30] Bochkareva E.S., Sidorov A.I., Yurina U.V., Podsvirov O.A. // Nucl. Instr. Meth. Phys. Research B. 2017. Vol. 403. P. 1-6.

[31] Рабинович В.А., Хавин 3.Я. Краткий химический справочник. Л.: Химия, 1978. 392 с.

[32] Bohren C.F., Huffman D.R. Absorption and Scattering of Light by Small Particles. NY: John Wiley \& Sons, 1983. $544 \mathrm{p}$.

[33] Климов В.В. Наноплазмоника М.: Физматлит, 2009. 480 с. [Klimov V.V. Nanoplasmonics. Singapore: Pan Stanford, 2014.]

[34] Gutierrez Y., Ortiz D., de la Osa R.A., Sanz J.M., Saiz J.M., Gonzalez F., Moreno F. // Препринт arXiv:1705.02248v1 [physics.optics] 5 May 2017.

[35] Ruppin R. // Opt. Comm. 2000. Vol. 182. P. 273-279.

[36] Воронцова Е.М., Гречушников Б.Н., Дистлер Г.И., Петров Н.П. Оптические материалы для инфракрасной техники. М.: Наука, 1965. 335 с.

[37] Palik E.D. Handbook of Optical Constants of Solids. Vol. 3. San Diego, USA: Academic press, 1998. 320 p.

[38] Inagaki T., Arakawa E.T., Birkhoff R.D., Williams M.W. // Phys. Rev. B. 1976. Vol. 13. P. 5610-5612.

[39] Naby M.H.E. // Zeitschrift fur Physik. 1963. Vol. 174. P. 269279.

[40] Vazquez-Vazquez C., Banobre-Lopez M., Mitra A., LopezQuintela M.A., Rivas J. // Langmuir. 2009. Vol. 25. P. 8208-8216.

[41] Лущик Ч.Б., Лущик А.Ч. Распад электронных возбуждений с образованием дефектов в твердых телах. М.: Наука, 1989. $263 \mathrm{c}$. 